\title{
Quality of Life in Patients with Urinary Incontinence: A Cross Sectional Study
}

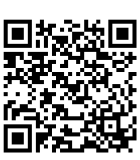

\author{
Lama Al Mehaisen* \\ Department Obstetrics and Gyn, Al-Balqa Applied University, Salt-Jordan
}

Submission: August 12, 2020; Published: September 21, 2020

*Corresponding author: Lama Al-Mehaisen, Md, MRCOG, Consultant Obstetrician and Gynecology, Associated Professor, Medical School, Obstetrics and Gyn Dept, Al-Balqa Applied University, Salt-Jordan

\section{Abstract}

Background: Urinary incontinence in women severely affects the physical, social and psychological aspects of life. Objectives: To find out the Quality of Life in female patients with urinary incontinence in a tertiary care hospital.

Materials and Methods: A cross-sectional, observational study was conducted in the Gynecology out-patient department. All non-pregnant women $\geq 21$ years of age, attending the Gynecology OPD of tertiary care hospital for various complaints were included in the study. After obtaining written informed consent, demographics, relevant medical and surgical history was noted, and they were then administered two validated questionnaire 'Incontinence Quality of Life Questionnaire' (IQOL) and SF-36.

Result: The overall incidence of incontinence in this population was $17.0 \%$, with a steadily rising incidence by age. The incidence was more in postmenopausal than premenopausal women. Menopausal women compared to non-menopausal women also had significantly higher incontinence QOL scores (median of 5 vs. 3; p=0.001). Avoidance and limiting behavior (ALB), Psychosocial impact (PSI) and social embarrassment (SE) scores were studied improved after 24 weeks. Items determining ALB had high correlation coefficient with total ALB and all of them were statistically significant. Items determining PSI had statistically significant correlation with mean PSI with all the items except two items. But among the items

Keywords: Female urinary incontinence; Incontinence quality of life questionnaire; SF36; Quality of life

\section{Introduction}

Process of urination is adapted in early childhood as an essential social behavior. Social and cultural norms demand that the act of urination be performed in private. Any degree of urine leakage or incontinence that compromises these norms results in discomfiture and initiates a social problem, as urine leakage may be associated with severe consequences on the mental wellbeing of the patient, feeling of alienation, depression or isolation, and may also adversely affect the personal and professional relations of patients. The International Continence Society (ICS) in the year 1976 defined incontinence as "the involuntary loss of urine that is a social hygienic problem". To be termed as incontinence, the urine leakage must typically occur in the absence of urinary tract infection. The terms 'social' and 'problem' in the definition highlight the compromise in quality of life that individuals who suffer from this problem must make. To facilitate survey, the ICS has provided a simpler definition of incontinence i.e., "the complaint of any involuntary loss of urine". As per this definition, more than half the women above 20 years of age have reported one or more episodes of incontinence [1]. The four main types of urinary incontinence are urge, stress, mixed, and functional incontinence. The clinical features the various types of incontinences somewhat overlap, but each type has certain discrete features. Urge incontinence is characterized by the involuntary leakage of urine accompanied by or immediately preceded by urgency. Stress incontinence is associated with urine leakage that occurs because of increased abdominal pressure from laughing, sneezing, coughing, climbing stairs, or other physical stressors on the abdominal cavity and, thus, the bladder. It is the most common type of urinary incontinence in younger women, but the incidence is highest in women between 45 and 49 years old. Mixed incontinence is a Urinary Incontinence combination of urge and stress incontinence, marked by involuntary leakage associated with urgency and with exertion, effort, sneezing, or coughing. Functional incontinence is the inability to hold urine due to reasons other than neuro-urologic and lower urinary tract dysfunction (eg, delirium, psychiatric disorders, urinary infection, reduced mobility) [2]. 
Because of anatomical difference like short urethra, more chances of recurrent UTI and because of pregnancy-childbirth or hysterectomy related injuries; urinary incontinence is more prevalent in women. Urinary incontinence is estimated to affect 200 million people worldwide. The exact prevalence of urinary incontinence in women is not available because of underreporting. But it is estimated to be as high as 55\%.3 Urinary incontinence is associated with disturbance in day-to-day activities, sleep, sexual functions and causes psychological problems. There are multiple facets of urinary incontinence that have the potential to affect health-related quality of life, because both evaluation and treatment may alter quality of life. The disorder may affect emotional and social facets and may also have an impact on activities of daily living and role fulfillment. Because there is a lack of data on exact prevalence and quality of life in female patients with urinary incontinence, we decided to conduct a cross sectional survey about Quality of life in female patients with urinary incontinence in a tertiary care hospital.

\section{Urinary Incontinence}

\section{Ethics}

Ethics Committee permission was obtained prior to commencement of the study. Written informed consent was obtained from all the women prior to their inclusion in the study.

\section{Study population}

All non-pregnant women $\geq 21$ years of age, attending the Gynecology OPD of King Abdullah University Hospital for various complaints were included in the study. After obtaining written informed consent, demographics, relevant medical and surgical history was noted. They were then administered a validated questionnaire 'Incontinence Quality of Life Questionnaire' (IQOL) by the same medical person. SF-36, a commonly used general QOL questionnaire was also administered to the patients concurrently with IQOL.

\section{IQOL Questionnaire}

The IQOL was a patient reported questionnaire. It was divided into 3 subscales with a total of 22 items pertaining to the symptoms of urinary incontinence:
a) Avoidance and limiting behavior (ALB)
b) Psychosocial impact (PSI)
c) Social embarrassment (SE)

\section{Scoring by IQOL}

Each item was to be scored on a 5-point Likert scale of 0 (not at all) to 4 (extremely). A mean score for each subscale is calculated (averaging the scores for the items in each subscale) as well as a total score for all 22 items (sum of all subscale scores). The scores were then transformed to a 'Scale score' ranging from 0-100 points for ease of interpretation: Scale score $=$ (sum of the items - lowest possible score)/possible raw score range X 100 Urinary Incontinence.

\section{Interpretation of IQOL}

For all items, higher scores indicated less impact of urinary incontinence on quality of life. MCID: MID (Minimally Important Difference) was approximately 4 points when defined as that corresponding to a small effect size ( $0.2 \mathrm{SD}$ at baseline) and approximately 11 points when defined as corresponding to a medium effect size (0.5 SD at baseline). SEM: ranged from 8-11 points. The IQOL questionnaire was translated into Arabic language and depending upon the language that the woman was best comfortable with, either English or Arabic questionnaire was provided.

\section{Statistical analysis}

Categorical variables were described using frequencies and percentages; continuous variables were described using range, mean and standard deviation (SD) if normal, and range, median, and inter-quartile range (IQR) if non-normal. Comparison of groups and categorical variables (cross127 tabulation) was conducted using chi square if the variable was binomial or multinomial and gamma/Kendall's tau b if ordinal. Comparison of continuous variables (non-normal) was conducted using Mann-Whitney $\mathrm{U}$ test. An $\alpha$ of 0.05 was considered statistically significant. All statistical analysis was conducted using IBM SPSS Statistics 19.0 (IBM, Chicago, IL) using 2- tailed tests. Sample size No formal sample size was calculated for this study. All non-pregnant women visiting the Gynecology OPD were sequentially enrolled in the study.

\section{Result}

The overall incidence of incontinence in this population was $17.0 \%$, with a steadily rising incidence by age. The differences are statistically significant (Kendall's tau b: 0.264 ; gamma $=0.546$; $\mathrm{p}=4.1 \times 10-12139$ ). The incidence of incontinence was more than twice as much in menopausal women compared to non-menopausal women $(27.2 \%$ vs. $12 \%$; $=8.0 \times 10-6140)$. patients out of 555 were postmenopausal .49 patients out of the 180 had urinary incontinence (27.2\%) 375 patients were still menstruating; 45 patient only complained of urinary incontinence (12\%). Total QOL incontinence score for all subjects (excluding "how do you feel about yourself" and patient reporting of QOL because these are reverse scales compared to all other questions) was a non-normal variable: median: 3.0; range: 0-66 (maximum possible score=76); IQR (inter-quartile range): 10 . There was a highly statistically significant difference between incontinence QOL score depending on whether subjects had incontinence or not: median: 22 vs. 2 ( $n=90$ and 446); $\mathrm{p}=4.6 \times 10-32$. Although there was a trend toward a lower incontinence QOL score for those subjects aged 30 years or less, the differences between groups were not significant. Menopausal women compared to non-menopausal women also had significantly higher IQOL scores (median of 5 vs. 3; $\mathrm{p}=0.001$ ). However, when analysis was restricted to those subjects with incontinence, there was no difference in IQOL score (median=22) for those women 


\section{Global Journal of Reproductive Medicine}

who were menopausal versus non-menopausal. Patient reported QOL score was not statistically different regarding presence or absence of incontinence: median $=2$, mean $=2.17$; no incontinence: median=2, mean=2.35). Likewise, there was even less difference by menopause status (data not shown).

However, respondents scored quite differently when they were asked whether they felt good about themselves; for incontinent subjects their mean/median scores were $1.87 / 2.0$ compared to non-incontinent subjects whose scores were 2.31/3.0. This difference was statistically significant $(\mathrm{p}=0.003)$. Fifty two out of 94 women who had urinary incontinence were assessed for the ALB, PSI and SE due to urinary incontinence. The results are provided respectively. When ALB due to urinary incontinence was assessed, the item 'I have difficulty getting a good night's sleep because of my incontinence' had the maximum mean of 1.79, with SD of 1.14 and the item 'I worry about coughing/sneezing because of my incontinence' had the least mean of 1.5 with SD of 0.828 . When PSI due to urinary incontinence was assessed, the item 'My incontinence makes me feel helpless' had the maximum mean of 1.83 with SD of 1.279 and item 'I get less enjoyment out of life because of my incontinence' had the least mean of 1.42 with SD of 0.801 . When SE due to urinary incontinence was assessed, it was observed that the item 'I worry about being embarrassed or humiliated by my incontinence' had the highest mean of 1.96 with SD of 1.427 and item 'I feel I have no control over my bladder' had the least mean of 1.6 with SD of 0.934 . Correlation between individual items and total scores of ALB, PSI, SE and total QOL) the items determining ALB had high correlation coefficient with total ALB and all of them were statistically significant. Among the items determining PSI, all the items except two items had statistically significant correlation with mean PSI. But among the items determining SE, two items had no significant relationship with SE. Total QOL had significant relationship with all the items except one item of PSI and one item of SE. On comparison of IQOL mean scores with different domains of SF36 questionnaire, role physical had significantly positive relation with ALB, role emotional had significantly negative relation with PSI and SE had a significant and positive relation with bodily pain but negative and significant relationship with general health and vitality.

\section{Discussion}

There is an increasing prevalence of bladder control problems as the world population ages. Simultaneously, there is increasing attention to maintaining an active lifestyle. Treatment of urinary incontinence includes options such as pelvic floor exercises, vaginal devices, oral medications, peri-urethral bulking agents, botox injections into the bladder, and surgery. The medical and surgical management available for the treatment of urinary incontinence poses an economic burden, with poor patient satisfaction $[3,4]$. This further adds to the poor quality of life in patients. The Fourth ICI has stressed the importance of initial assessment of quality of life in patients of urinary incontinence [5]. Most patients with urinary incontinence decide to seek treatment due to the adverse impact of incontinence on the QOL. Disease specific QOL questionnaires provide a standard method of assessing the impact of symptoms of urinary incontinence on QOL of patients. Urinary incontinence is more common in women than in men, due to more exposure to pelvic trauma during childbirth. It is two times more common in women than in men [2]. Age is the single largest risk factor for urinary incontinence. An important finding of this study is that the incidence of incontinence showed a steady rise with age and was higher in menopausal women. This corroborates with the epidemiology of urine where the prevalence is reported to be $6.9 \%$ in women aged $20-39$ years, $17.2 \%$ in those aged $40-59$ years, $23.3 \%$ in those aged $60-79$ years, and $31.7 \%$ in women older than 80 years of age.6The results of patient reported QOL suggest that while subjects may not feel that their quality of life is impacted by incontinence when asked to assess quality of life, but it does nevertheless affect them in terms of how good they feel about themselves. The IQOL is a highly used and widely recommended scale. Among other populations, the scale has been shown to be reliable, valid, and responsive to change. No ceiling effects have been reported. The questionnaire is easy to understand and poses little respondent burden. However, the assessment cannot be completed by proxy [4].

Our study provides symptom specific quality of life outcomes for urinary incontinence. The 3 domains of IQOL viz. ALB, PSI and SE showed that urinary incontinence affects the QOL of patients [6]. These effects could be especially bothersome in younger patients where incontinence has been shown to severely affect the emotional behavior and recreation and pastimes in younger women [7]. Moreover, it has been found that women with moderate to severe urinary incontinence may develop clinical depression requiring drug treatment [8]. In our study, we found that, barring a few, most items of IQOL individually correlated with the total subscale/domain scores, and with the overall QOL. The items of SF-36 correlated poorly with the domains of IQOL, indicating that a disease specific questionnaire must be used to assess the health related QOL in patients with symptoms of urinary incontinence. Also, IQOL may be used in future to measure the care provided to women with urinary incontinence and to assess treatment satisfaction. Our study assessed the women patients at a single center, whereas larger multicentric studies would provide substantial evidence of the impact of incontinence on QOL. To conclude, urinary incontinence affects many women and adversely affects the quality of life. Because of the lack of selfreporting, detection is inadequate. Disease specific self-reported questionnaires can help assess the impact of symptoms of urinary incontinence on QOL.

\section{References}

1. Abrams $P$ (2003) Impact of stress urinary incontinence on quality of life. Advanced studies in Medicine 3(8E): S829-S833.

2. Vasavada SP (2014) Urinary incontinence 
3. Wood LN, Anger JT (2014) Urinary incontinence in women. BMJ 349: g4531.

4. (2013) Incontinence Quality of Life Questionnaire.

5. Abrams P, Cardozo L, Khoury S, Wein A (2009) Incontinence, ( $4^{\text {th }} 245$ edn.), Health Publication Ltd., Paris.

6. Nygaard I, Barber MD (2008) Prevalence of Symptomatic Pelvic Floor Disorders in US Women. JAMA 300(11): 1311-1316.

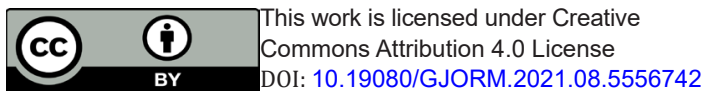

7. Hunskaar S, Vinsnes A (1991) The quality of life in women with urinary incontinence as measured by the sickness impact profile. J Am Geriatr Soc 39(4): 378-382.

8. Melville JL, Delaney K, Newton K, Katon W (2005) Incontinence severity and major depression in incontinent women. Obstet Gynecol 106(3): 585-592.

Your next submission with Juniper Publishers
will reach you the below assets
- Quality Editorial service
- Swift Peer Review
- Reprints availability
- E-prints Service
- Manuscript Podcast for convenient understanding
- Global attainment for your research
- Manuscript accessibility in different formats
( Pdf, E-pub, Full Text, Audio)
- Unceasing customer service
Track the below URL for one-step submission
https://juniperpublishers.com/online-submission.php

